\title{
The effect of different type la supernova progenitors on Galactic chemical evolution
}

\author{
F. Matteucci ${ }^{1,2}$, E. Spitoni ${ }^{1}$, S. Recchi ${ }^{3}$, and R.Valiante ${ }^{4}$ \\ 1 Dipartimento di Astronomia, Universitá di Trieste, via G. B. Tiepolo 11, 34143 Trieste, Italy \\ e-mail: matteucc@oats.inaf.it \\ 2 INAF Osservatorio Astronomico di Trieste, via G. B. Tiepolo 11, 34143 Trieste (TS), Italy \\ 3 Institute of Astronomy, Vienna University, Türkenschanzstrasse 17, 1180, Vienna, Austria \\ ${ }^{4}$ Dipartimento di Astronomia, Universita di Firenze, Largo E. Fermi 5, 50125 Firenze, Italy
}

Received 17 February 2009 / Accepted 7 April 2009

\begin{abstract}
Aims. Our aim is to show how different hypotheses about type Ia supernova progenitors can affect Galactic chemical evolution. Supernovae Ia are believed to be the main producers of $\mathrm{Fe}$ and the timescale with which Fe is restored into the interstellar medium depends on the assumed supernova progenitor model. This is a way of selecting the most appropriate progenitor model for supernovae Ia, a still debated issue.

Methods. We include different type Ia SN progenitor models, identified by their distribution of time delays, in a very detailed chemical evolution model for the Milky Way which follows the evolution of several chemical species. We test the single degenerate and the double degenerate models for supernova Ia progenitors, as well as other more empirical models based on differences in the time delay distributions.

Results. We find that assuming the single degenerate or the double degenerate scenario produces negligible differences in the predicted $[\mathrm{O} / \mathrm{Fe}]$ vs. $[\mathrm{Fe} / \mathrm{H}]$ relation. On the other hand, assuming a percentage of prompt (exploding in the first $100 \mathrm{Myr}$ ) type Ia supernovae of 50\%, or that the maximum type Ia rate is reached after 3-4 Gyr from the beginning of star formation, as suggested by several authors, produces more noticeable effects on the $[\mathrm{O} / \mathrm{Fe}]$ trend. However, given the spread still existing in the observational data, no model can be firmly excluded on the basis of only the $[\mathrm{O} / \mathrm{Fe}]$ ratios. On the other hand, when the predictions of the different models are compared with the G-dwarf metallicity distribution, the scenarios with very few prompt type Ia supernovae can be excluded. Conclusions. Models including the single degenerate or double degenerate scenario with a percentage of 10-13\% of prompt type Ia supernovae produce results in very good agreement with the observations. A fraction of prompt type Ia supernovae larger than $30 \%$ worsens the agreement with observations and the same occurs if no prompt type Ia supernovae are allowed. In particular, two empirical models for the type Ia SN progenitors can be excluded: the one without prompt type Ia supernovae and the one assuming a delay time distribution that is $\propto t^{-0.5}$. We conclude that the typical timescale for the Fe enrichment in the Milky Way is around 1-1.5 Gyr and that type Ia supernovae already should appear during the halo phase.
\end{abstract}

Key words. Galaxy: evolution - stars: supernovae: general - Galaxy: solar neighbourhood

\section{Introduction}

The type Ia supernova (SN) rate, which depends on the SN Ia progenitor model and the star formation history, is a fundamental ingredient in models of galactic chemical evolution. The progenitor model can be described simply by a delay time distribution (DTD) function, which is the distribution of the explosion times. In the pioneering work of Greggio \& Renzini (1983a, hereafter GR83) there was, for the first time, an expression for the type Ia $\mathrm{SN}$ rate in the scenario of the single degenerate model. In this scenario, $\mathrm{SNe}$ type Ia arise from the explosion of a $\mathrm{C}-\mathrm{O}$ white dwarf in a close binary system where the companion is either a red giant or a main sequence star (Whelan \& Iben 1973; Munari \& Renzini, 1992; Kenyon et al. 1993; Hachisu et al. 1996, 1999; Han \& Podsiadlowski 2004). The DTD of this model arises from the above described scenario and predicts the explosion times of binary systems with the appropriate characteristics. An alternative model for progenitors of type Ia SNe was proposed by Iben \& Tutukov (1984). In this scenario, two $\mathrm{C}-\mathrm{O}$ white dwarfs merge after loss of angular momentun due to gravitational wave emission, and explode because the final object reaches the Chandrasekhar mass. Tornambé \& Matteucci (1986) formulated a type Ia SN rate in this scenario and applied it to galactic chemical evolution models. Subsequently, Matteucci \& Greggio (1986) tested the GR83 rate by means of a detailed chemical evolution model of the Milky Way and interpreted the $[\alpha / \mathrm{Fe}]$ ratios versus $[\mathrm{Fe} / \mathrm{H}]$ as due to the delay in the Fe production from type Ia SNe, thus confirming previous suggestions (Tinsley 1979; Greggio \& Renzini 1983b). In recent years other authors (Dahlen et al. 2004; Strolger et al. 2004; Mannucci et al. 2005, 2006; Scannapieco \& Bildsten 2005; Sullivan et al. 2006; Aubourg et al. 2008; Pritchet et al. 2008; Totani et al. 2008) have proposed type Ia SN rates based on DTDs derived empirically. One example is the rate of Mannucci et al. (2005, 2006): they suggested that two populations of progenitors of type Ia $\mathrm{SNe}$ are needed to explain the dependence of the rates on the colors of the parent galaxy. The presence of type Ia SNe in old, red, quiescent galaxies is an indication that part of these $\mathrm{SNe}$ originate from old stellar populations. On the contrary, the increase of the rate in blue galaxies 
(by a factor of 30 going from $(B-K) \sim 2$ to $(B-K) \sim 4.5$ ) shows that some of the SN Ia progenitors are related to young stars and closely follow the evolution of the star formation rate (hereafter SFR). Mannucci et al. (2006, hereafter MVP06), on the basis of the previously described relation between the type Ia SN rate and the color of the parent galaxies, their radio power as measured by Della Valle et al. (2005), and cosmic age, concluded that there are two populations of progenitors of type Ia SNe. They have suggested (but see Greggio et al. 2008) that the current observations can be accounted for only if about half of the SNe Ia (prompt SNe Ia) explode within $10^{8}$ years after the formation of their progenitors, while the rest explode during a wide period of time extending up to 10 Gyrs (tardy SNe Ia). Matteucci et al. (2006) applied this formulation of the type Ia SN rate to chemical evolution models of galaxies of different morphological type. They concluded that a fraction of 50\% of prompt type Ia SNe worsens the agreement with abundance data, especially in the Milky Way, and suggested that prompt type Ia SNe should indeed exist but their fraction should not be larger than $30 \%$. They also found a possible scenario for SN Ia progenitors in the MVP06 framework: a possible justification for this strongly bimodal DTD can be found in the framework of the single degenerate model for the progenitors of type Ia SNe. In fact, such a DTD can be found if one assumes that the function describing the distribution of mass ratios inside the binary systems is a multi-slope function. In particular, this choice means that in the range 5-8 $M_{\odot}$ the systems where $M_{1} \sim M_{2}$ are preferred, whereas for lower mass progenitor systems where $M_{1} \gg M_{2}$ are favored. Another suggestion came from Dahlen et al. (2004) and Strolger et al. (2004, 2005), on the basis of the observational cosmic type Ia SN rate. They suggested a DTD with no prompt type Ia SNe with a maximum occurring at 3-4 Gyr. This DTD has never been tested in chemical evolution models. More recently, a direct determination of the DTD function has been reported by Totani et al. (2008), on the basis of the faint variable objects detected in the Subaru/XMM-Newton Deep Survey (SXDS). They concluded that the DTD function is inversely proportional to the delay time, i.e. the DTD can be well described by a featureless power law (DTD $\propto t^{-n}$, with $n \sim 1$ ). A similar suggestion came from Pritchet et al. (2008), who suggested a DTD $\propto t^{-0.5 \pm 0.2}$.

The maximum at which the the type Ia SN rate occurs is a very important parameter which affects the shape of the $[\alpha / \mathrm{Fe}]$ relation in galaxies. This timescale depends on the fraction of prompt/tardy type Ia SNe and on the specific history of star formation in galaxies, as shown by Matteucci \& Recchi (2001), being shorter than in the solar neighbourhood for ellipticals and longer for irregular systems.

In this paper, we show the effects of adopting different DTDs in a model of the chemical evolution of the Milky Way: in particular, we will focus on three relevant observational costraints: i) the $[\mathrm{O} / \mathrm{Fe}]$ vs. $[\mathrm{Fe} / \mathrm{H}]$ relation in the solar vicinity for which much accurate data are now available; ii) the type Ia SN rate at the present time and iii) the G-dwarf metallicity distribution. To do this, we adopt five different DTDs, three of which are empirically derived. The effects of the single degenerate and double degenerate DTDs has already been tested in previous papers, as for example in De Donder \& Vanbeveren (2004), where detailed close binary evolution models were included in the Chiappini et al. (1997) chemical evolution model; here we will explore also the effects of DTDs empirically derived in recent years.

The paper is organized as follows: in Sect. 2 the formulation for the SNIa rates is presented. In Sect. 3 the galactic chemical evolution model is described and the results are discussed, and in Sect. 4 some conclusions are drawn.

\section{Type la supernovae}

\subsection{Progenitors}

We recall here the most common models for the progenitors of type Ia SNe proposed this far:

- The merging of two C-O white dwarfs (WDs), due to gravitational wave radiation, which reach the Chandrasekhar mass $\left(\sim 1.4 M_{\odot}\right)$ and explode by C-deflagration (Iben \& Tutukov 1984). This is known as the double-degenerate (DD) scenario. In the original paper of Iben \& Tutukov the progenitor masses are in the range of 5-9 $M_{\odot}$ to ensure two WDs of at least $\sim 0.7 M_{\odot}$ in order to reach the Chandrasekhar mass. In Greggio (2005, hereafter G05) the range of progenitors is $2.0-8.0 M_{\odot}$, so that different combinations of WD masses are allowed, although always leading them to exceed the Chandrasekhar mass. The timing of the explosion in this scenario is given by the lifetime of the secondary plus the gravitational time delay.

- The C-deflagration of a Chandrasekhar mass C-O WD after accretion from a non-degenerate companion (Whelan \& Iben 1973; Munari \& Renzini 1992; Kenyon et al. 1993). This model is known as the single-degenerate (SD) one. The main problem with this scenario is the narrow range of permitted values of the mass accretion rate in order to obtain a stable accretion, instead of an unstable accretion with a consequent nova explosion and mass loss. In this case, in fact, the WD never achieves the Chandrasekhar mass. In particular, Nomoto et al. (1984) found that a central carbondeflagration of a WD is obtained for a high accretion rate $\left(\dot{M}>4 \times 10^{-8} M_{\odot} \mathrm{yr}^{-1}\right)$ from the secondary to the primary star (the WD). They found that $\sim(0.6-0.7) M_{\odot}$ of Fe plus traces of elements from $\mathrm{C}$ to $\mathrm{Si}$ are produced in the deflagration, well reproducing the observed spectra. The clock for the explosion here is given by the lifetime of the secondary star.

- A sub-Chandrasekhar C-O WD exploding by He-detonation induced by accretion of He-rich material from a He star companion (Tornambé \& Matteucci 1987; Limongi \& Tornambé 1991).

- A further model by Hachisu et al. $(1996,1999)$ is based on the classical scenario of Whelan \& Iben (1973), but they find an important metallicity effect. When the accretion process begins, the primary star (WD) develops an optically thick wind which helps in stabilizing the mass transfer process. When the metallicity is low $([\mathrm{Fe} / \mathrm{H}]<-1)$, the stellar wind is too weak and the explosion cannot occur. The clock for the explosion here is also given by the lifetime of the secondary star. However, one has to take into account that type Ia SN progenitor systems do not form before the gas has attained a threshold metallicity. Therefore, the chemical enrichment from type Ia SNe is delayed because of this effect.

\subsection{The assumed DTDs}

We have tested the effects of five DTDs:

- The Matteucci \& Recchi (2001, hereafter MR01) one which is practically the same as GR83, and is based on the single degenerate model for type I SN progenitors (see Matteucci et al. 2006, for details). In this DTD the fraction of prompt type Ia $\mathrm{SNe}$ is $13 \%$. This formulation has proven one of the best to describe the chemical evolution of most galaxies. The first $\mathrm{SNe}$ Ia to explode in this scenario are systems made up of an $8+8 M_{\odot}$ which takes $\sim 30-35 \mathrm{Myr}$. 
- The DTD suggested by G05 for the DD scenario: in particular, the DTD characterized by the exponent of the distribution function of final separations $\beta_{\mathrm{a}}=-0.9$, by the exponent of the gravitational delay time distribution $\beta_{\mathrm{g}}=-0.75$ and by a maximum nuclear delay time (lifetime of the secondary star) $\tau_{n, x}=0.4$ Gyr. The reason for chosing these parameters resides in the fact that they ensure a not too flat distribution of separations, which seems to be required to reproduce the specific type Ia SN rate in galaxies as a function of color (see Greggio \& Cappellaro 2009). In this DTD the fraction of prompt type Ia SNe is $10 \%$. The very first SNe Ia to explode in this scenario are systems made up of an $8+8 M_{\odot}$ which takes $\sim 30-35$ Myr plus the minimum gravitational time delay (1 Myr, G05).

- The DTD of MVP06. Here the fraction of prompt type Ia $\mathrm{SNe}$ is 50\%. The first systems also explode after 30-35 Myr.

- The DTD proposed by Strolger et al. (2004, hereafter S04) with a maximum at 3-4 Gyr. Here the first systems explode after $2.5 \times 10^{8}$ years. Therefore, there are no prompt type Ia SNe.

- The DTD proposed by Pritchet et al. (2008, PHS08), with DTD $\propto t^{-0.5}$, where the fraction of prompt type Ia SNe is $\sim 4 \%$. The one proposed by Totani et al. (2008) proceeds as $t^{-1}$ and we did not test it since these authors have already shown that it is very similar to the DTD of G05 for the DD scenario.

In Fig. 1 we show the different DTDs including those mentioned above. As one can see, while the DTDs of the SD and DD scenarios are similar, those of MVP06 and S04 and PHS08 are quite different. We did not take into account the Hachisu et al. (1996, 1999) model since its effects were already discussed in Kobayashi et al. (1998) and MR01. We did not also consider the sub-Chandra model because the correponding $\mathrm{SN}$ rate was computed by Tornambé \& Matteucci (1987). Finally, we show the DTD relative to the DD model (close channel) but we will not use it to compute galactic chemical evolution, as it is very similar to the DD (wide channel) DTD.

\subsection{The calculation of the type la $S N$ rate}

Once a DTD has been assumed, the type Ia SN rate is computed according to Greggio (2005):

$R_{\mathrm{Ia}}(t)=k_{\alpha} \int_{\tau_{i}}^{\min \left(t, \tau_{x}\right)} A(t-\tau) \psi(t-\tau) D T D(\tau) \mathrm{d} \tau$

where $\psi(t)$ is the SFR and $A(t-\tau)$ is the fraction of binary systems which give rise to type Ia SNe; in principle it can vary over time. Here we will assume $A$ to be a constant. The fraction A represents the fraction of binary systems with those particular characteristics that give rise to type Ia $\mathrm{SNe}$ relative to the whole range of star masses $\left(0.1-100 M_{\odot}\right)$. The time $\tau$ is the delay time defined in the range $\left(\tau_{i}, \tau_{x}\right)$ so that:

$\int_{\tau_{i}}^{\tau_{x}} D T D(\tau) \mathrm{d} \tau=1$

where $\tau_{i}$ is the minimum delay time for the occurrence of type Ia $\mathrm{SNe}$, in other words the time at which the first SNe Ia start occurring, and $\tau_{x}$ is the maximum delay time. Clearly these delay times vary according to the assumed progenitor scenario: in particular, in the SD scenario $\tau_{x}$ is the maximum nuclear delay time, namely the lifetime of the smallest secondary star, whereas in the

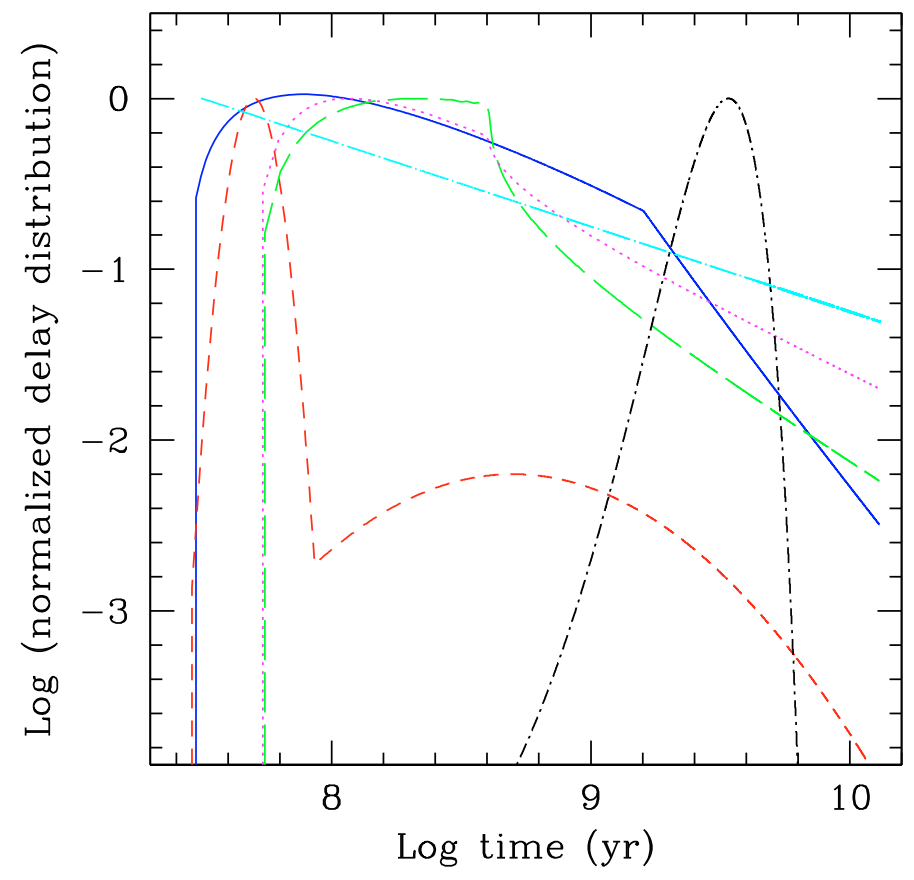

Fig. 1. Various DTD functions normalized to their own maximum value: the continuous blue line is the DTD of MR01; the long dashed green line is the DTD of G05 for the DD wide channel; the dotted magenta line is the DTD for the DD close channel of G05; the dashed red line is the DTD of MVP06; the short dashed-dotted black line is the DTD of S04, the cyan long dashed-dotted line is the DTD of PHS08.

DD model it is the sum of the maximum nuclear delay time and the maximum gravitational delay time.

Finally, $k_{\alpha}$ is the number of stars per unit mass in a stellar generation and contains the IMF.

In particular:

$k_{\alpha}=\int_{m_{L}}^{m_{U}} \phi(m) \mathrm{d} m$

with the normalization condition for the IMF being:

$\int_{m_{L}}^{m_{U}} m \phi(m) \mathrm{d} m=1$

where $m_{L}=0.1 M_{\odot}$ and $m_{U}=100 M_{\odot}$ and define the whole range of existence of the stars.

\section{The model for the Milky Way}

The model we adopt to compute the chemical evolution of the Milky Way is based on the original two-infall model by Chiappini et al $(1997,2001)$. They assumed that the first infall episode was responsible for the formation of the halo and thickdisk stars that originated from a fast dissipative collapse. The second infall episode formed the thin-disk component, with a timescale much longer than that of the thick-disk formation. The timescale for the formation of the halo-thick disk is 1-2 Gyr, while the timescale for the thin disk is $7 \mathrm{Gyr}$ in the solar vicinity. The authors also included in the model a threshold in the gas density, below which the star formation process stops. The existence of such a threshold value is suggested by observations relative to the star formation in external disk galaxies (Kennicutt 1998; but see Boissier et al. 2007). The physical reason for a threshold in the star formation is related to the gravitational stability, according to which, below a critical density, the gas is 
stable against density condensations and, consequently, the star formation is suppressed.

In the two-infall model, the halo-thick disk and the thin disk evolutions occur at different rates and they are independent, mostly as a result of different accretion rates. With these precise prescriptions it is possible to reproduce the majority of the observed properties of the Milky Way and this shows how important the choice of the accretion law is for the gas coupled to the SFR in the Galaxy evolution. Some of the most important observational constraints are represented by the various relations between the abundances of metals $(\mathrm{C}, \mathrm{N}, \alpha$-elements, iron peak elements) as functions of the $[\mathrm{Fe} / \mathrm{H}]$ abundance (the most common tracer of metallicity) and by the G-dwarf metallicity distribution. The Galactic disk is approximated by a series of concentric annuli, $2 \mathrm{kpc}$ wide, without exchange of matter between them. In this model, the thin disk forms "inside-out", in the sense that the timescale for the disk formation increases with galactocentric distance. This choice was dictated by the necessity of reproducing the abundance gradients along the Galactic disk (see Chiappini et al. 2001).

The time-evolution of the fractional mass of the element $i$ in the gas within a galaxy, $G_{i}$, is described by the basic equation:

$\dot{G}_{i}=-\psi(t) X_{i}(t)+R_{i}(t)+\left(\dot{G}_{i}\right)_{\text {inf }}-\left(\dot{G}_{i}\right)_{\text {out }}$

where $G_{i}(t)=M_{\mathrm{g}}(t) X_{i}(t) / M_{\mathrm{tot}}$ is the gas mass in the form of an element $i$ normalized to a total fixed mass $M_{\text {tot }}$ and $G(t)=$ $M_{\mathrm{g}}(t) / M_{\mathrm{tot}}$ is the total fractional mass of gas present in the galaxy at the time $t$. To model spiral disks, the masses are substituted by the surface mass densities. The quantity $X_{i}(t)=$ $G_{i}(t) / G(t)$ represents the abundance by mass of an element $i$, with the summation over all elements in the gas mixture being equal to unity.

$R_{i}(t)$ represents the returned fraction of matter in the form of an element $i$ that the stars eject into the ISM through stellar winds and supernova explosions; this term contains all the prescriptions concerning the stellar yields and the supernova progenitor models.

The assumed SFR $(\psi(t))$ is a Schmidt (1955) law with a dependence on the surface gas density $(k=1.5$, see Kennicutt 1998) and also on the total surface mass density (see Dopita \& Ryder 1994). In particular, the SFR is based on the law originally suggested by Talbot \& Arnett (1975) and then adapted by Chiosi(1980):

$\psi(r, t)=v\left(\frac{\Sigma(r, t) \Sigma_{\mathrm{gas}}(r, t)}{\Sigma\left(r_{\odot}, t\right)^{2}}\right)^{(k-1)} \Sigma_{\mathrm{gas}}(r, t)^{k}$

where $t$ is the time, $r$ is the galactocentric distance and the constant $v$ is the efficiency of the star formation process and is expressed in $\mathrm{Gyr}^{-1}$ : in particular, $v=2 \mathrm{Gyr}^{-1}$ for the halo and $1 \mathrm{Gyr}^{-1}$ for the disk $(t \geq 1 \mathrm{Gyr})$. The total surface mass density is represented by $\Sigma(r, t)$, whereas $\Sigma\left(r_{\odot}, t\right)$ is the total surface mass density at the solar position, assumed to be $r_{\odot}=8 \mathrm{kpc}$ (Reid 1993). The quantity $\Sigma_{\text {gas }}(r, t)$ represents the surface gas density. These choices of values for the parameters allow the model to fit the observational constraints very well, in particular in the solar vicinity. A threshold gas density for the star formation in the thin disk of $7 M_{\odot} \mathrm{pc}^{-2}$ is adopted in all the models presented here. The SFR described by Eq. (6) is equivalent to a simple Kennicutt's law $\left(S F R=v \Sigma_{\text {gas }}^{k}\right)$ concerning the evolution of chemical abundances, as shown in Colavitti et al. (2009). We have here used the formulation of Eq. (6) for consistency with our previous papers. The IMF is that of Scalo (1986) normalized over a mass range of $0.1-100 M_{\odot}$ and it is assumed to be constant in space and time.
The nucleosynthesis prescriptions are common to all models and are taken from Woosley \& Weaver (1995) yields for massive stars (those of Fe relative to the solar chemical composition and those of $\mathrm{O}$ metallicity dependent, since this is the best combination to reproduce the observations, as shown by François et al. 2004); van den Hoeck \& Groenewegen (1997) metallicity dependent yields for low and intermediate mass stars $\left(0.8 \leq M / M_{\odot} \leq 8\right)$ and Nomoto et al.'s (1997) yields for type Ia $\mathrm{SNe}$. The choice of these particular yields resides in the fact that they give the best agreement with the observed abundances in the solar vicinity, as shown by François et al. (2004). Moreover, the $\mathrm{O}$ yields are those best known, since the majority of nucleosynthesis studies agree on this particular element. The yields of Fe from massive stars are still more uncertain and they vary from author to author: however, most of the Fe is assumed to be produced by type Ia SNe. This is known from observational studies, which suggest that these $\mathrm{SNe}$ produce, on average, $0.5 M_{\odot}$ of Fe (see Blanc \& Greggio 2008, and references therein). The assumed stellar lifetimes for all galaxies are those suggested by Padovani \& Matteucci (1993).

The two terms $\left(\dot{G}_{i}\right)_{\text {inf }}$ and $\left(\dot{G}_{i}\right)_{\text {out }}$ account for the infall of external gas and for galactic winds, respectively. For the Milky Way we assume no wind, therefore $\left(\dot{G}_{i}\right)_{\text {out }}=0$.

The infall, instead, is an important physical process in the Galaxy. We assume the two-infall law of Chiappini et al. (1997):

$A(r, t)=a(r) \mathrm{e}^{-t / \tau_{\mathrm{H}}(r)}+b(r) \mathrm{e}^{-\left(t-t_{\max }\right) / \tau_{\mathrm{D}}(r)}$

where $a(r)$ and $b(r)$ are two parameters fixed by reproducing the total present time surface mass density along the Galactic disk. This particular infall law has proven to be consistent with an accretion law derived in a cosmological context, by assuming that gas accretion follows the accretion of the dark matter halo for a galaxy like the Milky Way (Colavitti et al. 2008). Colavitti et al. (2008) tested various infall laws including cosmologically derived ones and constant and linear laws. These different infall laws influence the SFR which depends on the amount of gas present in the galaxy at any time, and therefore the chemical abundances and the stellar metallicity distribution. They concluded that the best law in order to fit the main properties of the Milky Way is still Eq. (7) which has also been adopted in other chemical evolution studies (e.g. Chang et al. 1999; Alibès et al. 2001).

In the solar vicinity the total surface mass density $\sigma_{\text {tot }}=$ $51 \pm 6 M_{\odot} \mathrm{pc}^{-2}$ (see Boissier \& Prantzos 1999). $t_{\max }=1.0 \mathrm{Gyr}$ is the time for the maximum infall on the thin disk, $\tau_{\mathrm{H}}=2.0 \mathrm{Gyr}$ is the time scale for the formation of the halo thick-disk and $\tau_{\mathrm{D}}(r)$ is the timescale for the formation of the thin disk and it is a function of the galactocentric distance (inside-out formation, Matteucci \& François 1989; Chiappini et al. 2001).

In Fig. 2 we show the star formation rate as predicted by Eq. (6) in the framework of the two-infall model. Note the gap in the star formation rate at about $1 \mathrm{Gyr}$; this corresponds to the transition phase between the halo-thick disk and the thin disk phase. The gap is due to the fact that the disk is formed by means of the second infall episode and star formation can occur only if the gas density is above the threshold. Therefore, the gap occurs as a natural consequence of the two infall episides and a threshold gas density for star formation. Our chemical results would not be much affected if we had considered only one infall episode and no threshold in the gas density. In this case, the SFR would be simply exponential without oscillations and there would be no gap between the thick and thin disk. The $[\mathrm{O} / \mathrm{Fe}]$ vs. $[\mathrm{Fe} / \mathrm{H}]$ (see next section) would differ only for the lack of a loop 


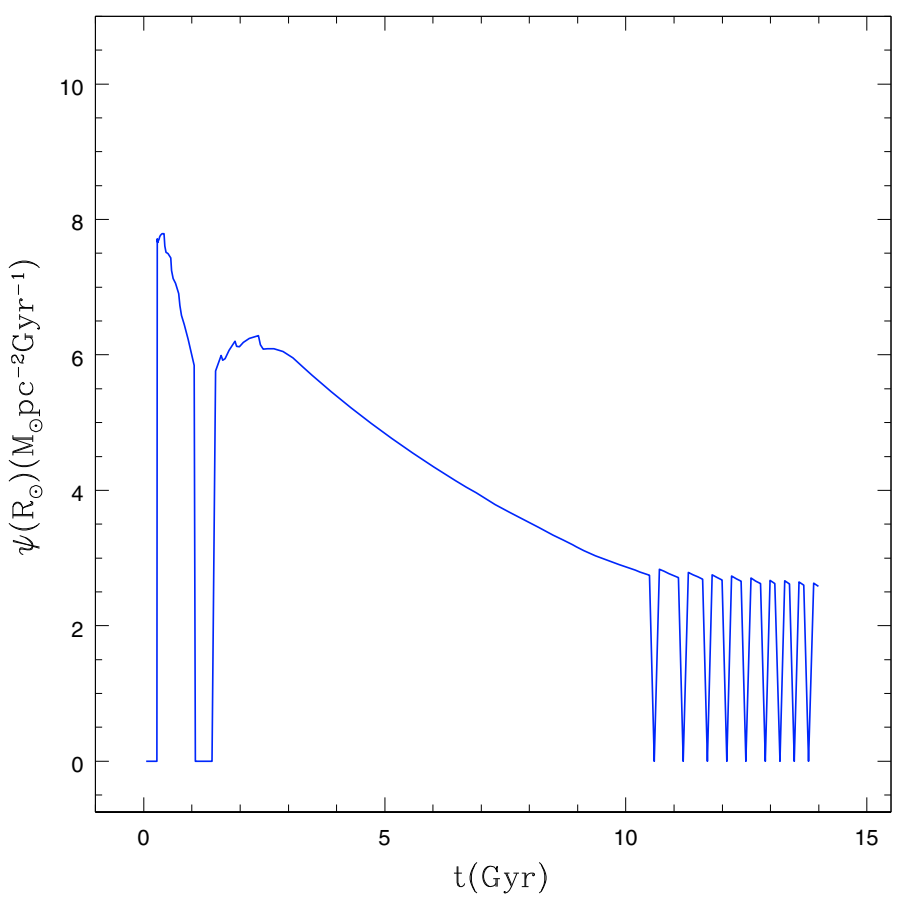

Fig. 2. The star formation rate as predicted by the two-infall model in the solar neighbourhood, the first peak represents the star formation rate during the halo-thick disk phase. Note that there is a short gap in the star formation between the end of the thick disk phase and the beginning of the thin disk phase: this is due to the adoption of a threshold density for the star formation. Note that the oscillating behaviour in the last few Gyr is due to the fact that the threshold density is easily reached at late times and star formation stops for a while until the gas restored by dying stars again increases the interstellar gas density.

in the transition phase between thick and thin disk (see for example Matteucci \& François 1989, 1992), as it is instead predicted by the two-infall model.

\section{Predicted $[\mathrm{O} / \mathrm{Fe}]$ vs. $[\mathrm{Fe} / \mathrm{H}]$ with differents $\mathrm{DTDs}$}

In Fig. 3 we show the predicted type Ia $\mathrm{SN}$ rates for the solar vicinity in the framework of the two-infall model previously described and for the considered DTDs. We have chosen the parameter $A$ in Eq. (1) in order to reproduce the present time type Ia $\mathrm{SN}$ rate in the Galaxy $\left(\sim 0.3 \mathrm{SNe}\right.$ century ${ }^{-1}$, Cappellaro et al. 1999). A value of $A \sim 0.0025$ for all the DTDs was chosen, in agreement with Matteucci et al. (2006), with the exception of the PHS08 DTD for which the parameter $A=0.0002$. The PHS08 DTD predicts the largest fraction of SNe Ia exploding with large delays (see Fig. 1). That means that a larger fraction of long-living progenitors (born several Gyr ago, when the SFR was much higher, see Fig. 2), relative to the other models, is exploding at the present time. Therefore, a smaller value of $A$ is required to reproduce the present-time $\mathrm{SN}$ Ia rate in the Galaxy. The oscillating behaviour shown by some type Ia SN rates in the last few Gyr is clearly related to the oscillations in the star formation rate. In particular, models with a larger percentage of prompt type Ia $\mathrm{SNe}$ show oscillations at late times (SD model and MVP06 model), whereas the models of S04, G05 and PHS08, having null, $10 \%$ and $4 \%$ fractions of prompt type Ia $\mathrm{SNe}$, respectively, show negligible oscillations.

The effect of the gap in the star formation rate, as predicted by the two-infall model, is evident for the DTDs of MVP06, SD, DD and PHS08 models. For the DTD of S04, there is no

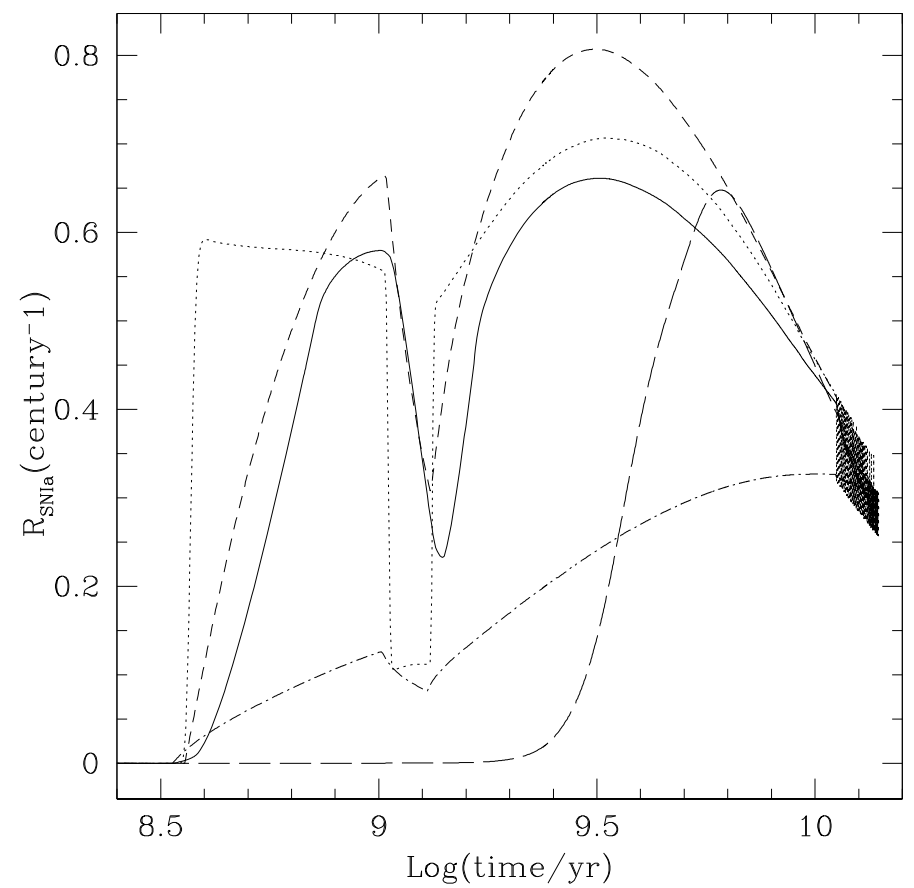

Fig. 3. The predicted type Ia SN rates in the solar vicinity in the framework of the two-infall model. The five curves refer to different DTDs. In particular, the dotted line represents the DTD of MVP06; the continuous line represents the SD model with the DTD from MR01 and the short dashed line the DD model with the DTD of G05 for the DD wide channel. Finally, the long dashed line represents the DTD suggested by S04 and the dashed-dotted line the DTD of PHS08. The rates are expressed in SN per century, and are all normalized to reproduce the present time type Ia SN rate in the Milky Way.

gap, since the minimum delay in the occurrence of type Ia SNe is much longer than for the other DTDs. There are no prompt type Ia SNe and most of the SNe Ia start occurring only after the gap in star formation. In Figs. 4-6 we show the effect of different DTDs on the $[\mathrm{O} / \mathrm{Fe}]$ vs. $[\mathrm{Fe} / \mathrm{H}]$ diagram. As it is well known, this diagram is interpreted on the basis of the "time-delay model". The time delay model suggests that the $[\mathrm{O} / \mathrm{Fe}]$ in the halo stars $([\mathrm{Fe} / \mathrm{H}]<-1.0)$ is slowly varying with $[\mathrm{Fe} / \mathrm{H}]$ and reflects mainly the behaviour of the $\mathrm{O} / \mathrm{Fe}$ ratio in massive stars; therefore, one expects a slight variation with the stellar mass. For $[\mathrm{Fe} / \mathrm{H}]>-1.0$, the $[\mathrm{O} / \mathrm{Fe}]$ ratio starts declining as a consequence of the increase of the Fe production from type Ia SNe. Clearly, the DTD is fundamental in determining the moment, and therefore the $[\mathrm{Fe} / \mathrm{H}]$ value, at which this process becomes important. This effect does not depend on details of the star formation history but mainly on the time delay between the chemical enrichment from SNe II and SNe Ia. On the basis of this, it is therefore important to see which DTD best fits the abundance data. In Fig. 4 we show the results for the DTDs related to the SD and DD (wide channel) models. As one can see, the differences between the results obtained by means of the SD and DD models are small, as expected from the comparison of the DTDs connected to these scenarios (see also Valiante et al. 2009). In particular, the amount of prompt type Ia SNe in the SD and DD scenarios is very similar, namely $13 \%$ and $10 \%$, as opposed to the DTD of MVP06 where the percentage of prompt SNe Ia is $50 \%$. This difference is evident in the $[\mathrm{O} / \mathrm{Fe}]$ ratios predicted by the MVP06 DTD (Fig. 5) which decline before and faster than for the SD and DD models. In the model with the MVP06 DTD, the $[\mathrm{O} / \mathrm{Fe}]$ knee occurs at $[\mathrm{Fe} / \mathrm{H}] \sim-1.8 \mathrm{dex}$, whereas for the 


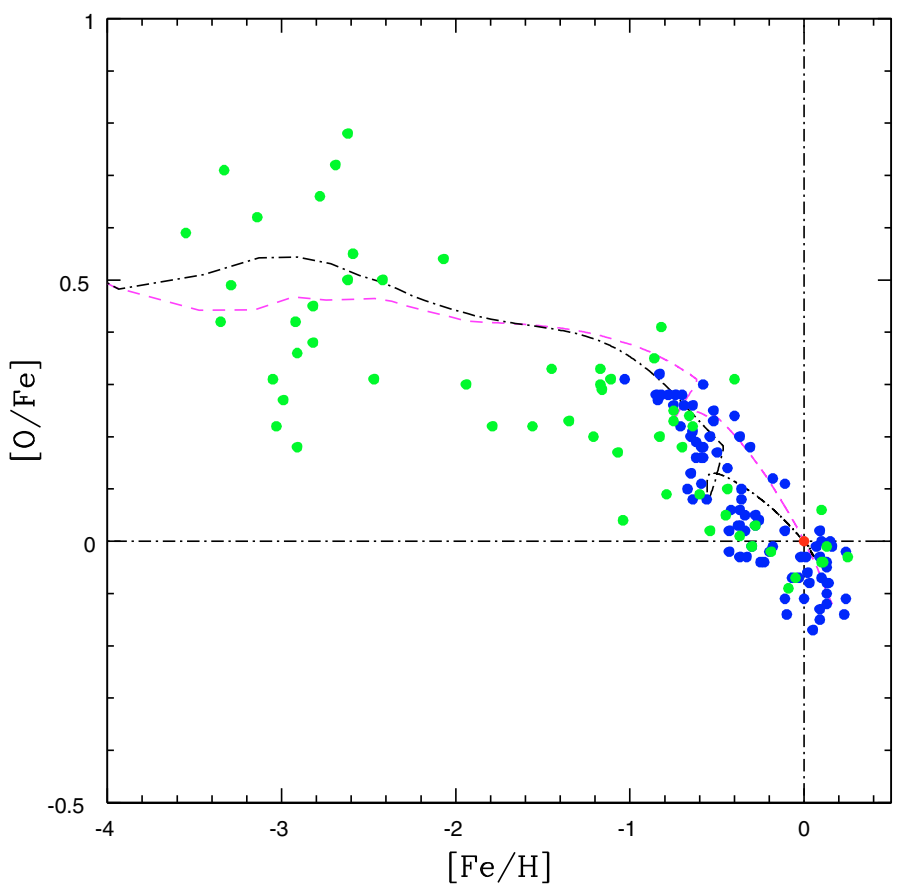

Fig. 4. The predicted $[\mathrm{O} / \mathrm{Fe}]$ vs. $[\mathrm{Fe} / \mathrm{H}]$ in the solar vicinity for two different DTDs: dashed line (DTD of SD model from MR01), dasheddotted (DTD of G05 for the wide DD channel). The model results are normalized to their own predicted solar values. The data are taken from the compilation of François et al. (2004). The solar position is marked by a red dot.

Table 1. Predicted $[\mathrm{O} / \mathrm{Fe}]_{\odot}$ and $[\mathrm{Fe} / \mathrm{H}]_{\odot}$ values at the time of formation of the solar system plus the value of $[\mathrm{Fe} / \mathrm{H}]_{\text {knee }}$ at which the knee for each model occurs. Normalization to the solar values of Asplund et al. (2005).

\begin{tabular}{cccc}
\hline \hline Model & {$[\mathrm{O} / \mathrm{Fe}]_{\odot}$} & {$[\mathrm{Fe} / \mathrm{H}]_{\odot}$} & {$[\mathrm{Fe} / \mathrm{H}]_{\text {knee }}$} \\
\hline SD & 0.095 & -0.013 & -0.8 \\
DD & 0.095 & -0.038 & -0.8 \\
MVP06 & 0.07 & -0.003 & -1.8 \\
S04 & 0.06 & 0.01 & -0.6 \\
PHS08 & 0.22 & -0.16 & -0.45 \\
\hline
\end{tabular}

results obtained with the SD and DD DTDs the knee occurs at $[\mathrm{Fe} / \mathrm{H}] \sim-0.8 \mathrm{dex}$ (see Table1).

In Fig. 5 we show the predicted $[\mathrm{O} / \mathrm{Fe}]$ trend for the DTD suggested by $\mathrm{S} 04$ where the delay in the appearence of the first type Ia $\mathrm{SNe}$ is much longer than in the previous cases. As one can see from the figure, the $[\mathrm{O} / \mathrm{Fe}]$ ratio in this case shows a longer plateau than in the other models, and the knee in the $[\mathrm{O} / \mathrm{Fe}]$ ratio occurs at $[\mathrm{Fe} / \mathrm{H}] \sim-0.6 \mathrm{dex}$. The cosmic epoch at which the knee in the $[\mathrm{O} / \mathrm{Fe}]$ ratio occurs in the models with the $\mathrm{SD}$ and DD rates is $\sim 1 \mathrm{Gyr}$, whereas in the case of the Strolger et al. DTD this time is 4-5 Gyr. In Fig. 5 we also show a comparison between the results obtained with a DTD $\propto t^{-0.5}$ (PHS08) and the DTD of MVP06. As one can see, the PHS08 DTD produces results not very different from the SD model but it predicts a too low solar Fe abundance $\left([\mathrm{Fe} / \mathrm{H}]_{\odot}=-0.16 \mathrm{dex}\right)$ and a too high solar $[\mathrm{O} / \mathrm{Fe}]_{\odot}=0.22$ dex, as opposed to all the other models where the solar $[\mathrm{Fe} / \mathrm{H}]_{\odot}$ and $[\mathrm{O} / \mathrm{Fe}]_{\odot}$ are very close to zero (see Table 1 where we show the predicted solar ratios). Note that the model results are always normalized to their own predicted solar values.

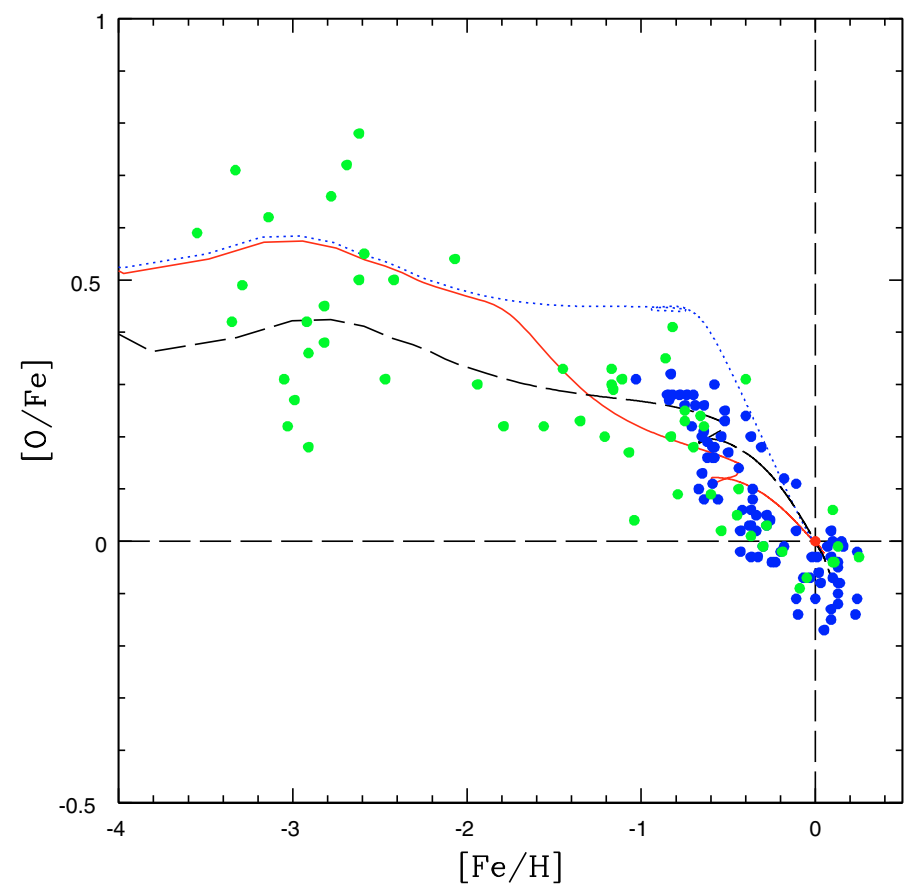

Fig. 5. Predicted $[\mathrm{O} / \mathrm{Fe}]$ when the Strolger et al. DTD is assumed (dotted line) compared to the predictions from the models with MVP06 DTS (continuous line) and the model with the DTD $\propto t^{-0.5}$ (PHS08) (long dashed line). The model results are normalized to their own predicted solar values. The data are the same as in Fig. 4.

In Fig. 6 we show a zoomed comparison of all the studied DTDs in the $[\mathrm{Fe} / \mathrm{H}]$ range $-1.5-0.5$ dex: here the different effects produced by the different assumptions about type Ia SN progenitors are more evident. A larger number of prompt type Ia $\mathrm{SNe}$ clearly produces lower $[\mathrm{O} / \mathrm{Fe}]$ ratios in this $[\mathrm{Fe} / \mathrm{H}]$ range than models decreasing the number of prompt $\mathrm{SNe}$ in favor of tardy ones. Although the spread existing in the data prevents us from drawing firm conclusions, from this comparison we see that the S04 model tends to predict too high values of $[\mathrm{O} / \mathrm{Fe}]$ in the range $-1.5 \leq[\mathrm{Fe} / \mathrm{H}] \leq-0.2$. On the other hand, the model with MVP06 DTD predicts the lowest $[\mathrm{O} / \mathrm{Fe}]$ ratios in the range $-1.8 \leq[\mathrm{Fe} / \mathrm{H}] \leq-1.0$. From numerical experiments, we can say that the MVP06 DTD with $\sim 30 \%$ prompt SNe would better fit the data, and it would still be compatible with the findings of MVP06. The DTDs influence the $[\mathrm{Fe} / \mathrm{H}]$ at which the gap in the star formation between the halo-thick disk and the thin disk phases is visible. This gap produces a loop in the $[\mathrm{O} / \mathrm{Fe}]$ behaviour and is naturally produced by assuming a threshold gas density in the star formation. Observationally, a gap in the abundance ratios has been claimed by Fuhrmann (1998) and Gratton et al. (2000). In particular, the gap was observed at a $[\mathrm{Fe} / \mathrm{H}]$ between -1.0 and $-0.5 \mathrm{dex}$, corresponding to the transition phase between the thick and the thin disk. If confirmed, this gap can impose constraints on the history of star formation. In our plots, the gap occurs at larger $[\mathrm{Fe} / \mathrm{H}]$ values going from the SD to PHS08, DD and MVP06 DTD (see Fig. 6). For the S04 DTD, the gap is not visible, as type Ia SNe start exploding in a non negligible number only after the gap. Therefore, the effect of the cessation of oxygen production, while the Fe is still produced by the type Ia SNe exploding during the gap, is lost. In the other models this effect produces the loop in the $[\mathrm{O} / \mathrm{Fe}]$ ratio. If the gap really exists, this would further strengthen the conclusion that prompt type Ia $\mathrm{SNe}$ should exist and be a non negligible fraction of all SNe Ia. 


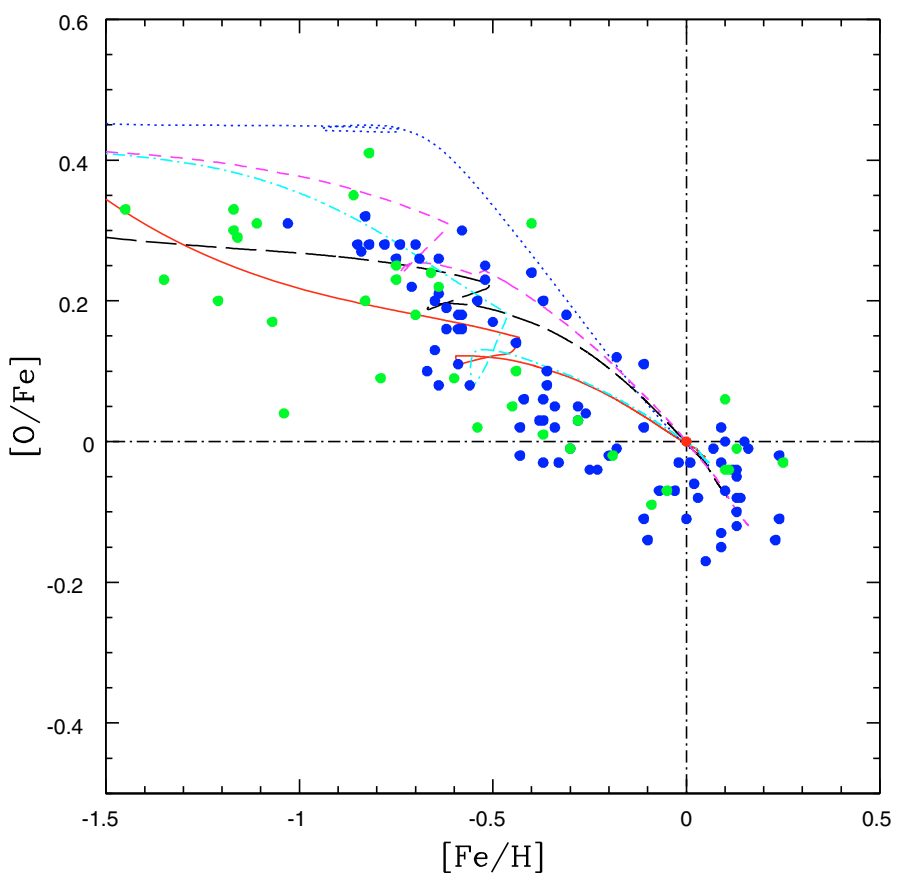

Fig. 6. Comparison of all the models with all the studied DTDs: the short dashed curve refers to the DTD of MR01; the continuous curve refers to the DTD of MVP06; the dotted line represents S04 DTD; the long dashed curve refers to the DTD of PHS08; the dashed-dotted curve refers to the DTD of the DD scenario (wide channel) of G05. The model results are normalized to their own predicted solar values. The data are the same as in Fig. 4.

In summary, the SD and DD models together with the bimodal distribution of MVP06 all produce acceptable results, although 50\% prompt type Ia SNe appears too large. The DTD from PHS08 and S04 are probably less likely, although the spread in the data prevents us from drawing firm conclusions.

Therefore, we checked our results on a very important observational constraint: the G-dwarf metallicity distribution. In Fig. 7 we show the predictions for the G-dwarf metallicity distribution of all our models compared with observational data. This figure shows that two models can be ruled out: the PHS08 and the S04. Both of these models contain empirically derived DTDs. They predict too many metal poor stars below $[\mathrm{Fe} / \mathrm{H}]<-1.0$ dex (the so-called G-dwarf problem), and this is due to the fact that in both cases the Fe abundance increases more slowly than in the other three models (SD, DD, MVP06), thus producing too many metal-poor stars. The reason for that is the small number of prompt type Ia SNe in the PHS08 and S04 models. In particular, the S04 model shows two distinct peaks in the G-dwarf metallicity distribution and this is due to the fact that at the beginning of the thin disk formation, only SNeII enrich the gas in $\mathrm{Fe}$ and the bulk of Fe comes with a large delay, thus creating the second peak. On the other hand, the other models all produce acceptable results, with the DD and MVP06 models being the best. Note that all the models predict less metal-rich stars than observed by Norström et al. (2004) which is the largest and more accurate survey of $\mathrm{G}$ and $\mathrm{F}$ dwarfs. This is a problem for pure models of chemical evolution which do not take into account the possibility of stellar migration. In fact, because of interaction between stars and transient spiral density waves (Roskar et al. 2008), stars born in the inner part of the disk can be scattered at larger galactocentric distances and this could be the explanation for the existence of metal-rich stars in the solar vicinity.

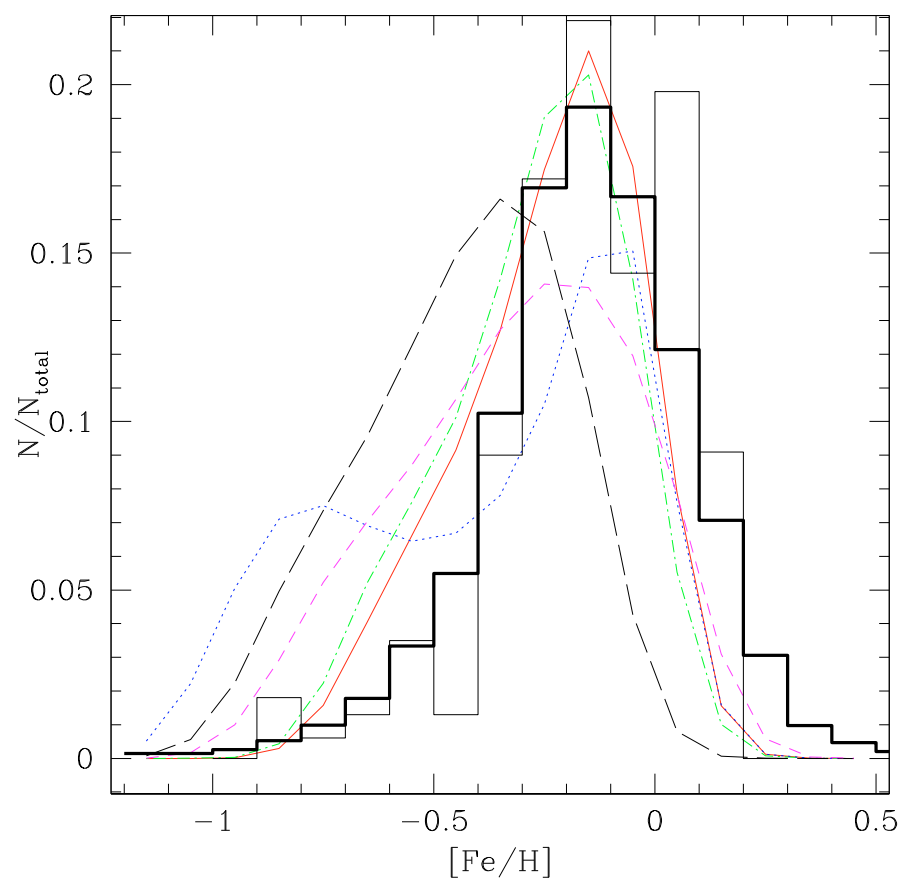

Fig. 7. The G-dwarf metallicity distribution in the solar vicinity. The data are from the Geneva-Copenhagen Survey of the Solar Neighbourhood, Nordstrom et al. (2004) (thick line histogram) and the data by Jorgensen (2000) (thin-line histogram). The models refer to: S04 DTD (dotted line), PSH08 DTD (long dashed line), DD model (dashed-dotted line), MVP DTD (continuous line) and SD model (short dashed line).

\section{Conclusions}

We have studied the effects of different DTDs on the $[\mathrm{O} / \mathrm{Fe}]$ vs. $[\mathrm{Fe} / \mathrm{H}]$ diagram, where the effects of the time delay in the Fe production by type Ia SNe are particularly important, as it is on the $\mathrm{G}$-dwarf-[Fe/H] distribution. We considered the DTDs related to the two main progenitor models for SNe Ia, namely the SD and DD models. Then we considered other DTDs derived empirically from the study of SN rates in galaxies (MVP06, PHS08) and the cosmic $\mathrm{SN}$ rate ( $\mathrm{S} 04)$, which are not directly related to particular progenitor models. We computed the chemical evolution of the solar vicinity by adopting a successful model of Galactic chemical evolution, the so-called two-infall model. Our main results can be summarized as follows:

- Delay time distributions containing a not negligible number of prompt type a $\mathrm{SNe}$ (namely those $\mathrm{SNe}$ exploding inside 100 Myr since the beginning of star formation) are necessary to best fit the $[\mathrm{O} / \mathrm{Fe}]$ ratio. This indicates that the first type Ia $\mathrm{SNe}$ occurring in galaxies can explode as soon as after 35-40 Myr since the beginning of star formation. This corresponds to the lifetime of an $8 M_{\odot}$ star, which is also the maximum mass for the progenitors of $\mathrm{C}-\mathrm{O}$ white dwarfs. As a consequence of this, the first type Ia $\mathrm{SNe}$ already appear during the halo phase.

- The bimodal DTD of MVP06 predicts a very high fraction of prompt type Ia $\mathrm{SNe}(\sim 50 \%)$. This fraction is probably too high since it depresses the $[\mathrm{O} / \mathrm{Fe}]$ ratio in the $[\mathrm{Fe} / \mathrm{H}]$ range $-1.8-0.8$ dex and produces a knee in the $[\mathrm{O} / \mathrm{Fe}]$ ratio at a too low metallicy (the observed knee is at $[\mathrm{Fe} / \mathrm{H}] \sim-1.0 \mathrm{dex}$ ). A smaller fraction of prompt SNe Ia would be acceptable (see also Matteucci et al. 2006). 
- The DTD of S04, which predicts the maximum of type Ia SNe occurring at 3.4 Gyr since the beginning of star formation, overproduces the $[\mathrm{O} / \mathrm{Fe}]$ ratio in the $[\mathrm{Fe} / \mathrm{H}]$ range $-1.0-0.2$ dex and predicts a knee in the $[\mathrm{O} / \mathrm{Fe}]$ ratio occurring at a too high $[\mathrm{Fe} / \mathrm{H}] \sim-0.6$ dex. Although the spread in the data prevents us from drawing firm conclusions, the best DTDs from the point of view of the $[\mathrm{O} / \mathrm{Fe}]$ diagram are the one related to the SD and DD cases.

- The G-dwarf metallicity distributions predicted by the models with different DTDs give a more clear indication of the DTDs which should be discarded; those are the two empirical ones derived by S04 and PSH08. They produce too many disk stars with metallicity $[\mathrm{Fe} / \mathrm{H}]<-1.0$ dex. Therefore, we conclude that the DTDs related to the two classical models for type Ia SN progenitors (SD and DD), and in particular the one related to the DD model (wide channel), are the best able to reproduce the observational data. Since these two models include prompt type Ia SNe even if in a smaller proportion than the bimodal DTD of MVP06, we conclude again that such prompt $\mathrm{SNe}$ are necessary to reproduce the chemical evolution of the solar vicinity. Probably a mixed scenario (SD+DD) would be the most realistic one (see De Donder \& Vanbeveren 2004; Greggio et al. 2008).

- On the basis of the results discussed above we can identify the typical timescale for Fe enrichment by type Ia SNe in the solar vicinity, identified by the time at which the knee in the $[\mathrm{O} / \mathrm{Fe}]$ ratio occurs, as $1-1.5 \mathrm{Gyr}$.

Acknowledgements. This work was partially supported by the Italian Space Agency through contract ASI-INAF I/016/07/0. F.M., E.S. and S.R. acknowledge financial support from PRIN2007-MUR (Italian Ministry of University and Research), Prot. 2007JJC53X_001. We also thanks an anonymous referee for his/her useful comments. S.R. acknowledges financial support from the FWF through the Lise Meitner grant M1079-N16.

\section{References}

Alibès, A., Labay, J., \& Canal, R. 2001, A\&A, 370, 1103 Asplund, M., Grevesse, N., \& Sauval, A. J. 2005, ASPC, 336, 25 Aubourg, E., Tojeiro, R., Jimenez, R., et al. 2008, A\&A, 492, 631 Blanc, G., \& Greggio, L. 2008, NewA, 13, 606

Boissier, S., Gil de Paz, A., Boselli, A., et al. 2007, ApJS, 173, 524 Boissier, S., \& Prantzos, N. 1999, MNRAS, 307, 857

Cappellaro, E., Evans, R., \& Turatto, M. 1999, A\&A, 351, 459

Chang, R. X., Hou, J. L., Shu, C. G., \& Fu, C. Q. 1999, A\&A, 350, 38 Chiappini, C., Matteucci, F., \& Gratton, R. 1997, ApJ, 477, 765

Chiappini, C., Matteucci, F., \& Romano, D. 2001, ApJ, 554, 1044
Chiosi, C. 1980, A\&A, 83, 206

Colavitti, E., Matteucci, F., \& Murante, G. 2008, A\&A, 483, 401

Colavitti, E., Cescutti, G., Matteucci, F., \& Murante, G. 2009, A\&A, 496, 429

Dahlen, T., Strolger, L. G., Riess, A. G., et al. 2004, ApJ, 613, 189

De Donder, E., \& Vanbeveren, D. 2004, New Astron. Rev., 48, 861

Della Valle, M., Panagia, N., Padovani, P., et al. 2005, ApJ, 629, 750

Dopita, M. A., \& Ryder, S. D. 1994, ApJ, 430, 163

François, P., Matteucci F., Cayrel R., et al. 2004, A\&A, 421, 613

Fuhrmann, K. 1998, A\&A, 338, 161

Gratton, R. G., Carretta, E., Matteucci, F., \& Sneden, C. 2000, A\&A, 358, 671

Greggio, L. 2005, A\&A, 441, 1055 (G05)

Greggio, L., \& Cappellaro, E. 2009, [arXiv:0902.0700]

Greggio, L., \& Renzini, A. 1983a, A\&A, 118, 217 (GR83)

Greggio, L., \& Renzini, A. 1983b, Mem. SaIt, 54, 311

Greggio, L., Renzini, A., \& Daddi, E. 2008, MNRAS, 388, 829

Hachisu, I., Kato, M., \& Nomoto, K. 1996, ApJ, 470, 97

Hachisu, I., Kato, M., \& Nomoto, K. 1999, ApJ, 522, 487

Han, Z., \& Podsiadlowski, P. 2004, MNRAS, 350, 1301

Jørgensen, B. R. 2000, A\&A, 363, 947

Kennicutt, R.C., Jr. 1998, ARA\&A, 36, 189

Kenyon, S. J., Livio, M., Mikolajewska, J., \& Tout, C. A. 1993, ApJ, 407, L81 Kobayashi, C., Tsujimoto, T., Nomoto, K., Hachisu, I., \& Kato, M. 1998, ApJ, 503, L155

Iben, I. Jr., \& Tutukov, A. 1984, ApJS, 54, 335

Limongi, M., \& Tornambé, A. 1991, ApJ, 371, 317

Mannucci, F., Della Valle, M., Panagia, N., et al. 2005, A\&A, 433, 807

Mannucci, F., Della Valle, M., \& Panagia, N. 2006, MNRAS, 370, 773 (MVP06)

Matteucci, F., \& François, P. 1989, MNRAS, 239, 885

Matteucci, F., \& François, P. 1992, A\&A, 262, L1

Matteucci, F., \& Greggio, L. 1986, A\&A, 154, 279

Matteucci, F., \& Recchi, S. 2001, ApJ, 558, 351 (MR01)

Matteucci, F., Panagia, N., Pipino, A., et al. 2006, MNRAS, 372, 265

Munari, U., \& Renzini, A. 1992, ApJ, 397, L87

Nomoto, K., Thielemann, F.-K., \& Yokoi, K. 1984, ApJ, 286, 644

Nomoto, K., Iwamoto, K., Nakasato, N., et al. 1997, NuPhA, 621, 467

Nordström, B., Mayor, M., Andersen, J., et al. 2004, A\&A, 418, 989

Padovani, P., \& Matteucci, F., 1993, ApJ, 416, 26

Pritchet, C. J., Howell, D. A., \& Sullivan, M. 2008, ApJ, 683, L25 (PHS08)

Reid, M. J., 1993, ARA\&A, 31, 345

Roskar, R., Debattista, V. P., Quinn, T. R., Stinson, G. S., \& Wadsley, J. 2008, ApJ, 684, L79

Scalo, J. M. 1986, FCPh, 11, 1

Scannapieco, E., \& Bildsten, L. 2005, ApJ, 629, L85

Schmidt, M. 1955, ApJ, 129, 243

Strolger, L. G., Riess, A. G., Dahlen, T., et al. 2004, ApJ, 613, 200 (S04)

Strolger, L. G., Riess, A. G., Dahlen, T., et al. 2005, ApJ, 635, 1370

Sullivan, M., Le Borgne, D., Pritchet, C. J., et al. 2006, ApJ, 648, 868

Talbot, R. J. Jr., \& Arnett, W. D. 1975, ApJ, 197, 551

Tinsley, B. M. 1979, ApJ, 229, 1046

Tornambé, A., \& Matteucci, F. 1986, MNRAS, 223, 69

Tornambé, A., \& Matteucci, F. 1987, ApJ, 318, L25

Totani, T., Morokuma, T., Oda, T., Doi, M., \& Yasuda, N. 2008, PASJ, 60, 1327

Valiante, R., Matteucci, F. Recchi, S., \& Calura, F. 2009, New Astron., 14, 638

van den Hoek, L. B., \& Groenewegen, M. A. T. 1997, A\&AS, 123, 305

Whelan, J., \& Iben, I., Jr. 1973, ApJ, 186, 1007

Woosley, S. E., \& Weaver, T. A. 1995, ApJS. 101, 181 